\title{
Salvage of Solitary Renal Autotransplant with Early Graft Thrombosis and Abdominal Mesh Closure
}

\author{
Morwan Bahi, MB ChB ${ }^{1 *}$, Lucy Guazzo, BSci MB BS MS'2, Dilip Naik, MB ChB FRACS ${ }^{1}$ and \\ Imad Aljanabi, MB ChB FRACS ${ }^{3}$ \\ ${ }^{1}$ Department of Vascular Surgery, Wellington Regional Hospital, Wellington, New Zealand \\ ${ }^{2}$ Department of Vascular Surgery, Royal Brisbane Hospital, Brisbane, Australia \\ ${ }^{3}$ Department of General Surgery, Wellington Regional Hospital, Wellington, New Zealand
}

\begin{abstract}
Fibromuscular dysplasia is a common cause of non-atherosclerotic renal artery stenosis in the young patient population leading to resistant hypertension. This is typically diagnosed radiologically and is often less responsive to endovascular revascularisation given the non-atherosclerotic nature of stenosis. As a result, surgery may be the only durable option for patients whereby hypertension is uncontrolled by medical management. Here, we will report the case of a young female with a solitary kidney affected by fibromuscular dysplasia and we will discuss the surgical management and complications including graft thrombosis and compartment syndrome requiring re-operation and use of mesh in abdominal wall closure.
\end{abstract}

Abbreviations

FMD: Fibromuscular Dysplasia; GSV: Great Saphenous Vein; RAS: Renal Artery Stenosis

\section{Introduction}

Fibromuscular dysplasia (FMD) refers to a group of conditions affecting arterial wall musculature resulting in non-atherosclerotic arterial stenosis [1]. This is a common underlying cause of renal artery stenosis (RAS) in young patients resulting in uncontrollable hypertension [1]. Given the non-atherosclerotic nature of RAS in this patient group, angioplasty is only mildly beneficial with short-term benefit [1]. As a result, many patients are referred for surgery in the form of renal autotransplantation or reconstruction of the stenosed renal artery [1].

Key causes of early transplantation failure are often surgical [2]. These include vascular thrombosis (arterial or venous) and retroperitoneal compartment syndrome secondary to elevated compartment pressure $[2,3]$. The latter can be a culprit for vascular thrombosis through kinking of vessels leading to interrupted blood flow and higher risk of thrombosis [2,3].

In this report, we discuss the case of a 30-year-old female requiring renal auto-transplantation for RAS caused by FMD with concomitant discussion of post-operative complications and operative decisions. The patient has provided permission for discussion of case details in this article.

\section{Case Report}

Our patient is a 30-year-old Caucasian female with left renal agenesis and a solitary $14 \mathrm{~cm}$ right kidney with malignant hypertension driven by right RAS secondary to underlying FMD. The patient's blood pressure was uncontrolled despite four anti-hypertensive agents and the patient did not show prolonged benefit from two attempts at endovascular revascularisation in the form of renal artery angioplasty. A consensus opinion was reached between the patient's treating nephrologist and transplant surgery team to offer right renal autotransplantation, excision of the stenotic renal artery segment and reconstruction with interposition graft. This was done using the patient's great saphenous vein (GSV) as a durable option for this patient's ongoing uncontrolled hypertension.

*Corresponding author: Morwan Bahi, MB ChB, Department of Vascular Surgery, Wellington Regional Hospital, Wellington, New Zealand

Accepted: June 11, 2021

Published online: June 13, 2021

Citation: Bahi M, Guazzo L, Naik D, et al. (2021) Salvage of Solitary Renal Autotransplant with Early Graft Thrombosis and Abdominal Mesh Closure. J Transplant Surg 4(1):72-74 
Following successful autotransplantation in the right pelvis via a retroperitoneal approach the kidney was noted to be well-perfused on closure and the patient made approximately $120 \mathrm{ml}$ of urine in the first hour. The kidney was large caliber and had to be carefully positioned to allow for closure of the abdominal wall. The patient underwent day zero ultrasound in recovery as per local protocol approximately $1.5 \mathrm{hrs}$ post closure. This showed thrombosis of the GSV interposition graft. The patient was immediately taken back to theatre for thrombectomy. The GSV interposition graft was found to contain thrombus which was evacuated successfully with a Fogarty catheter and the anastomosis was revised although no technical problem was identified. An attempt was then made to reposition the kidney into the abdominal cavity however closure without tension was not possible due to the size of the solitary kidney $(14 \mathrm{~cm})$. This was contributed to by oedema likely secondary to prolonged ischaemic time. The general surgical team attended theatre at this stage and the decision made to close the abdominal wall with mesh to release tension on the kidney. The external oblique aponeurosis was mobilised and a Symbotex composite mesh was used to reconstruct the external oblique layer allowing successful tension-free closure.

Post-operatively, the patient required a temporary period of dialysis and was eventually weaned off dialysis with improvement in renal function. More importantly, the patient's blood pressure normalized and she was able to cease all anti-hypertensive medications. The patient has not developed any complications with relation to the abdominal wall mesh closure on follow-up and continues to have a good outcome from this procedure with no evidence of complications on the six-month follow-up ultrasound.

\section{Discussion}

This is an interesting case for a number of reasons. This young patient with FMD-driven malignant hypertension also had left renal agenesis. The patient's solitary kidney was enlarged at $14 \mathrm{~cm}$ (see Figure 1) and this was a significant consideration in surgical planning. Any failure of autotranplantation in this patient would lead to complete loss of (previously normal) renal function due to her single kidney status. As a result of this atypically large kidney and the need for re-operation to thrombectomise the interposition graft, closure without tension was not possible and a composite mesh was used to reconstruct the abdominal wall (external oblique layer). This is not commonly described in renal transplantation literature and neither is retroperitoneal compartment syndrome.

Tension free abdominal wall muscle closure in renal transplantation is required due to risk of compartment syndrome resulting in compression of renal parenchyma or tension on vasculature resulting in kinking and potential risk of graft thrombosis $[4,5]$. In our case, the patient's large $(14 \mathrm{~cm})$ solitary kidney posed significant challenges with closure. Following artery reconstruction with GSV graft and autotransplantation, closure of the abdominal wall was difficult and required manipulation of the kidney into a position that allowed muscle approximation. However,

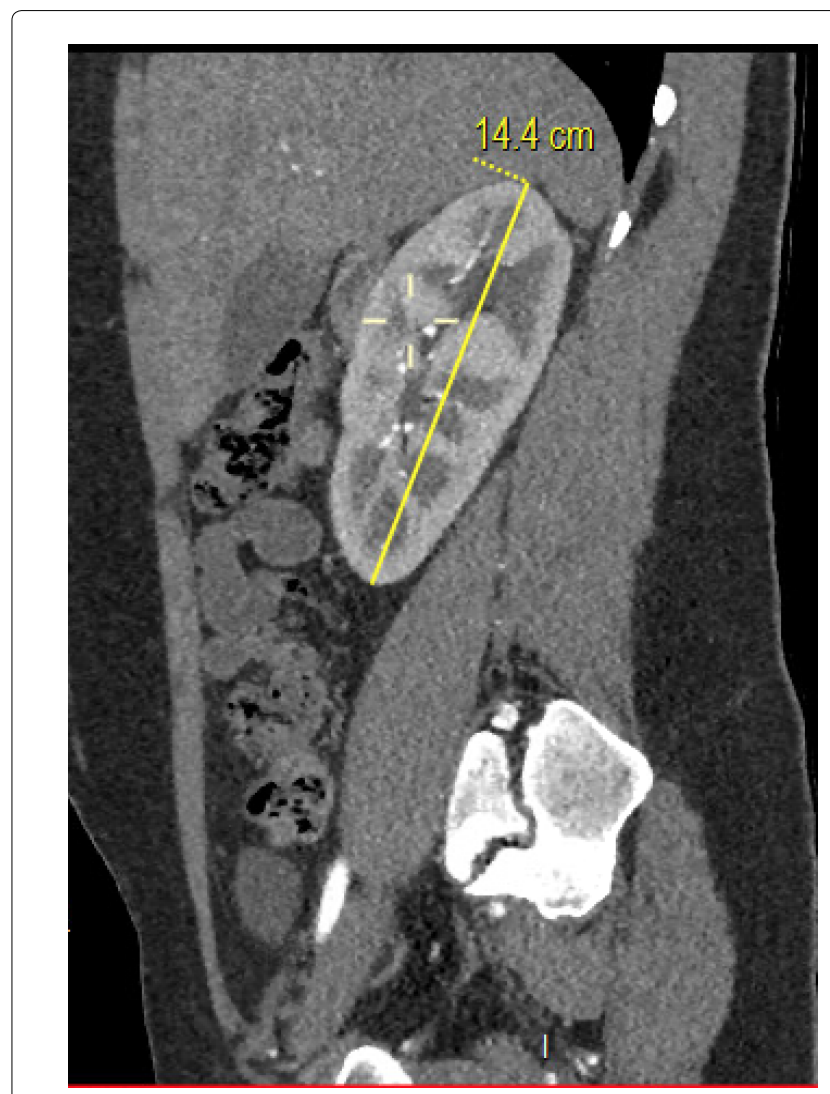

Figure 1: CT image showing large solitary right kidney.

in retrospect, this may have contributed tension onto the interposition graft. While difficult to ascertain retrospectively, it is possible the increased tension in the abdominal cavity may have led to graft kinking as a cause of thrombus formation due to interrupted flow. The abdominal wall closure was more difficult during the take-back operation likely secondary to renal oedema caused by prolonged warm ischaemic time [4] and the safest option was to opt for mesh closure.

We used a Symbotex composite mesh to close the external oblique muscle layer. This is a polyester synthetic mesh with a biodegradable collagen film on the backside (which prevents adherence to abdominal viscera) [6]. A $25 \times 20 \mathrm{~cm}$ Symbotex mesh was cut to size and secured to external oblique aponeurosis with running prolene suture and interrupted PDS. This allowed us to achieve tension-free closure in the takeback operation and has not resulted in any complication or sequelae in the immediate postoperative period or on our continued outpatient follow-up. There has been no evidence of hernia formation.

\section{Conclusion}

Solitary renal autotransplantation for renal artery stenosis secondary to fibromuscular dysplasia is not commonly described in the literature. Our case was further complicated by venous graft thrombosis requiring re-operation and difficulty achieving tension-free abdominal wall closure leading to reconstruction of the abdominal wall using mesh. This has been successful in management of the patient's hypertension and the patient continues to experience no complications secondary to the abdominal wall mesh closure. 


\section{References}

1. Trinquart L, Mounier-Vehier C, Sapoval M, et al. (2010) Efficacy of revascularisation for renal artery stenosis caused by fibromuscular dysplasia: A systematic review and meta-analysis. Hypertension 56: 525-532.

2. Phelan P, Magee C, O'Kelly P, et al. (2011) Immediate retransplantation following early kidney transplant thrombosis. Nephrology 16: 607-611.

3. Butt FK, Kokko KE, Araujo C, et al. (2012) Renal allograft compartment syndrome: An under-recognized cause of early dysfunction. Transplantation 94: 618.
4. Halawa A (2010) Abdominal wall closure of renal transplant recipients: An undermined challenge. Int J Organ Transplant Med 1: 73-76.

5. Beasley K, McAlister V, Luke P (2003) Mesh hood fascial closure in renal allograft compartment syndrome. Transplant Proc 35: 2418-2419.

6. Lepere M, Gillion J, Barrat C, et al. (2018) First year preliminary results on the use of a monofilament polyester mesh with a collagen barrier for primary and incisional ventral hernia repair. Int Surg 103: 56-65. 